\title{
Separation and Recovery of Nickel from Waste Electroless Nickel-Phosphorous Plating Solution
}

\author{
Tokie OOKUBO, Syouhei NISHIHAMA and Kazuharu YOSHIZUKA* \\ Department of Chemical Engineering, The University of Kitakyushu, \\ Hibikino 1-1, Kitakyushu 808-0135, Japan
}

(Received December 25, 2012; Accepted February 12, 2013)

\begin{abstract}
Separation and recovery of nickel from an actual waste solution of electroless nickel-phosphorous plating solution has been investigated, employing synergistic extraction with bis(2-ethylhexyl)phosphoric acid (D2EHPA) and nicotinic acid ester. The extractability of Ni with D2EHPA alone is low, due to suppression by phosphorous compounds coexisting in the waste solution. Effective extraction can be achieved with a synergistic extraction system, using both D2EHPA and nicotinic acid ester. By optimizing the operational conditions, satisfactory extraction and stripping of more than $95 \%$ yield can be achieved. The present extraction - stripping processing can be successfully repeated, although a small amount of the nicotinic compound is dissolved into the aqueous solution.
\end{abstract}

\section{Introduction}

Surface treatment is presently conducted by several methods, such as plating and deposition, to add required features. Plating is an electrochemical process having advantages of simple apparatus and an economical process. Electroless nickel (Ni) plating, including nickel-phosphorous (Ni-P) plating, is widely applied in the automobile and electronic industries, since electroless Ni plating has features of uniformity and is applicable to surfaces with micro structure. Plating of surfaces by electroless Ni-P plating is conducted by immersing substrates in a plating solution consisting of organic acids as complexing agents, hypophosphorous acid as a reducing agent, and nickel sulfate as a source of nickel. The plating rate is decreased by repeated use of the solution, due to the accumulation of impurities such as sulfate ion, phosphorous ion from the reducing agent, and zinc and/or iron, eluted from the plated surface. A large amount of waste plating solution containing ca. $2-8 \mathrm{~g} / \mathrm{L}$ of $\mathrm{Ni}$ is thus discharged, and most of the waste is treated by precipitation, followed by landfill. The amount of $\mathrm{Ni}$ in the waste solutions in Japan was 
estimated to be ca. 100 t/year in 2004 [1], and thus the recovery of Ni from the waste solution has been studied [2,3]. Okada et al. have reported on the extraction and stripping behavior of $\mathrm{Ni}$ from the electroless Ni plating waste solution by the synergistic extraction system of an organophosphoric acid and pyridine compounds, and found a high extraction ability for Ni from the waste liquor, although the pyridine compounds were lost to the aqueous solution [2]. A more detail investigation is therefore required to apply the synergistic extraction system for the separation and recovery of $\mathrm{Ni}$ from the waste solution, especially with regard to the extractants and the purity of Ni recovered.

In the present work, therefore, separation and recovery of $\mathrm{Ni}$ from an actual waste electroless Ni-P plating solution, obtained from a plating company has been investigated. The company possesses two electroless Ni-P plating lines, an electroless Ni line and an under barrier metal (UBM) line. From these 530 t/year of waste solution and $100 \mathrm{t} /$ year of sludge containing ca. $40 \mathrm{wt} \%$ of Ni, was discharged in 2010 . Separation and recovery of $\mathrm{Ni}$ from the waste plating solution can therefore contribute to the recycling of $\mathrm{Ni}$ from this waste and to a decrease in the amount of sludge. The compositions of two waste electroless Ni-P plating solutions from the electroless Ni line and the UBM line were firstly investigated. Solvent extraction was then applied to the waste solution. In the present work, synergistic extraction with an organophosphoric acid and pyridine compounds was employed to extract Ni quantitatively [2]. Optimization of the conditions for extraction and stripping was carried out. Finally the reusability of the extractant was investigated.

\section{Experimental}

Actual waste solutions of electroless Ni-P plating were used in the present work. The composition of the waste solutions was determined, as shown in Table 1, using an inductively coupled plasma atomic

Table 1. Composition of the waste electroless Ni-P plating solutions.

\begin{tabular}{|c|c|c|}
\hline \multirow{2}{*}{ Element } & \multicolumn{2}{|c|}{ Concentration (mg/L) } \\
\cline { 2 - 3 } & Electroless Ni line & UBM line \\
\hline $\mathrm{Na}$ & 6830 & 5.10 \\
$\mathrm{Si}$ & 11.0 & 0.526 \\
$\mathrm{P}$ & 48800 & 9140 \\
$\mathrm{Ca}$ & 35.1 & $\mathrm{~N}^{+} .^{\dagger}$ \\
$\mathrm{Ti}$ & N.D. $^{\dagger}$ & N.D. $^{\dagger}$ \\
$\mathrm{Fe}$ & 1.27 & 0.534 \\
$\mathrm{Ni}$ & 4080 & 5530 \\
$\mathrm{Cu}$ & 0.101 & 0.098 \\
\hline $\mathrm{pH}$ & ca. 4.0 & ca. 4.6 \\
\hline
\end{tabular}


emission spectrometer (ICP-AES, Shimadzu ICPS-7000). Bis(2-ethylhexyl)phosphoric acid (D2EHPA, Nacalai Tesque, Inc., Kyoto, Japan) and three types of pyridine compounds, nicotinic acid hexyl ester (NA-C6, Tokyo Chemical Industry Co., Ltd., Tokyo, Japan), nicotinic acid octyl ester (NA-C8, Tokyo Chemical Industry Co., Ltd., Tokyo, Japan), and 4-(3-phenylpropyl)pyridine (PP-P, Wako Pure Chemical Industries, Ltd., Osaka, Japan) were employed as extractants, and kerosene (Wako Pure Chemical Industries, Ltd., Osaka, Japan) was used as the diluent. All other reagents were supplied by Wako Pure Chemical Industries, Ltd.

Feed aqueous solutions were prepared by adding small amounts of $\mathrm{H}_{2} \mathrm{SO}_{4}$ or $\mathrm{NaOH}$ solutions to the waste solution to adjust the $\mathrm{pH}$ value, so that the change in the concentrations of the metals was negligible. Organic solutions were prepared by diluting D2EHPA only or combinations of D2EHPA and each pyridine compound having the same concentration in kerosene. Organic and aqueous solutions having an organic/aqueous volume ratio (O/A) of 1 were shaken for more than 24 hours at $298 \mathrm{~K}$. After phase separation, the equilibrium $\mathrm{pH}$ was measured. The concentrations of metal ions were determined by ICP-AES, and the extraction percentages were determined by material balance. The stripping behavior of the extracted $\mathrm{Ni}$ was also investigated. In this case, extraction of $\mathrm{Ni}$ was firstly carried out with $0.6 \mathrm{~mol} / \mathrm{L}$ D2EHPA/NA-C6 or D2EHPA/NA-C8 at an initial pH of 5.0, where the equilibrium pH was 4.3 and 3.3 for the electroless Ni line and the UBM line, respectively. The loaded organic solution was scrubbed with 0.01 $\mathrm{mol} / \mathrm{L} \mathrm{H}_{2} \mathrm{SO}_{4}$ solution at an $\mathrm{O} / \mathrm{A}$ ratio of 1 , followed by stripped with $\mathrm{H}_{2} \mathrm{SO}_{4}$ solutions of $0.2-0.8 \mathrm{~mol} / \mathrm{L}$ at an $\mathrm{O} / \mathrm{A}$ ratio of 1 .

\section{Results and Discussion}

\subsection{Extraction of Ni from waste electroless Ni-P plating solution with D2EHPA}

Extraction of $\mathrm{Ni}$ from the waste solution with D2EHPA was first investigated. The original waste solution from the UBM line was employed as the feed aqueous solution. The waste solution from the UBM line, 10-times diluted with deionized water, was also used as a feed solution for comparison, since the exact composition of the waste solution, such as the phosphorus compounds and the organic acids, was not clear. Figures $1 \mathrm{a}$ and $1 \mathrm{~b}$ show the effect of equilibrium $\mathrm{pH}$ on the extraction percentage and distribution ratio of $\mathrm{Ni}$ with D2EHPA. In both cases, extraction of $\mathrm{Ni}$ increases with increasing $\mathrm{pH}$ values due to the cation exchange mechanism, which has been widely reported [4,5]. When the waste solution without dilution was used, the $\mathrm{pH}$ range where extraction takes place is shifted to higher values, and the extraction percentage of $\mathrm{Ni}$ is quite low at all $\mathrm{pH}$ values investigated. In addition, the slope of $\log D$ against the equilibrium $\mathrm{pH}$ is smaller than 2, especially in the case of the original UBM line waste. A straightforward direct comparison cannot be carried out because the concentrations of the components in the feed solution are different. These differences are however likely to be due to the suppression of the extraction of $\mathrm{Ni}$ by anionic species in the waste solution, such as phosphorous compounds, and Ni complexes with the phosphorous compounds may be extracted into the organic phase. Effective extraction of Ni with D2EHPA alone is therefore difficult to 
achieve, even when the waste solution is diluted. The modification of the extraction system is therefore required to improve the extractability of $\mathrm{Ni}$ from the waste solution.
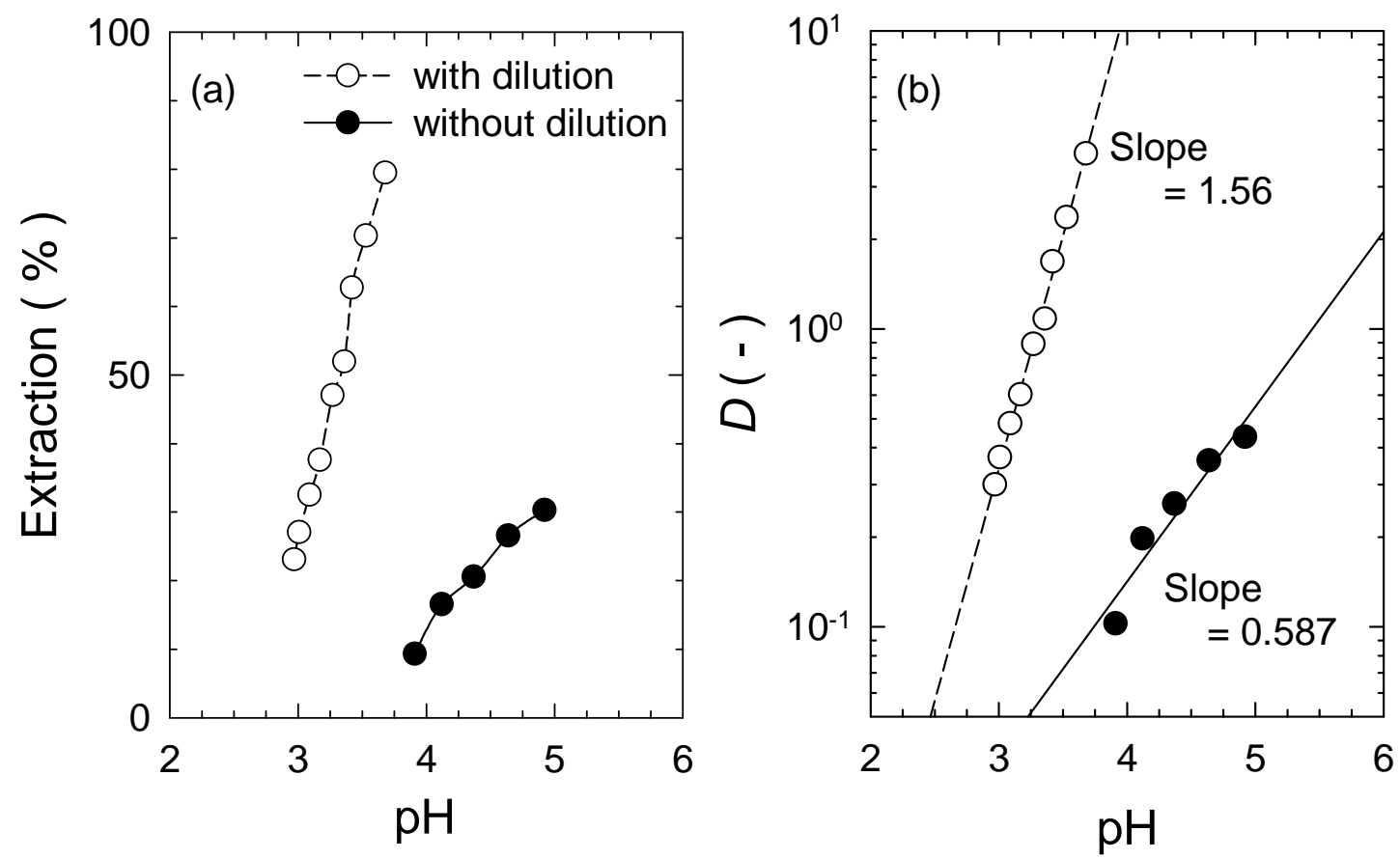

Figure 1. Effect of equilibrium $\mathrm{pH}$ on (a) extraction percentage and (b) distribution ratio of Ni with D2EHPA; $[\overline{\mathrm{D} 2 \mathrm{EHPA}}]=0.5 \mathrm{~mol} / \mathrm{L}$.

\subsection{Synergistic extraction of Ni from waste electroless Ni-P Plating solutions}

A synergistic extraction system has been reported to be effective in improving the extraction ability of Ni $[2,3,6]$. In the present work, combinations of D2EHPA and pyridine compounds were employed [2]. Figure 2 shows the effect of equilibrium $\mathrm{pH}$ on the extraction percentage of $\mathrm{Ni}$ from waste solutions with mixed extractants. In all cases, a synergistic effect for the extraction of $\mathrm{Ni}$ is observed, and thus the extraction percentage of $\mathrm{Ni}$ is dramatically improved compared with the extraction system with D2EHPA alone. This suggests that water molecules coordinated to Ni are replaced with the pyridine compounds [2]. For the electroless Ni line, the extraction percentage of Ni with D2EHPA/NA-C6 and D2EHPA/NA-C8 is almost the same and decreased at $\mathrm{pH}$ values $>4.5$, while that with D2EHPA/PP-P increased with $\mathrm{pH}$. The difference in the extraction behavior of Ni between the D2EHPA/NA-C6 and D2EHPA/NA-C8 systems and the D2EHPA/PP-P system may be attributed to both the competitive complexation of $\mathrm{Ni}$ with the phosphorous compounds in the waste solution and dissolution of PP-P into the aqueous solution, though the true reason is not clear at this moment. In the case of D2EHPA/PP-P, however, PP-P is likely to be dissolved in the aqueous phase, which indicates that the reuse of this extractant cannot be achieved. For the UBM line, the extraction percentage of $\mathrm{Ni}$ increased with increasing $\mathrm{pH}$ in all systems. The extraction 
percentage with D2EHPA/NA-C6 and D2EHPA/NA-C8 is almost the same, and is higher than that with D2EHPA/PP-P.

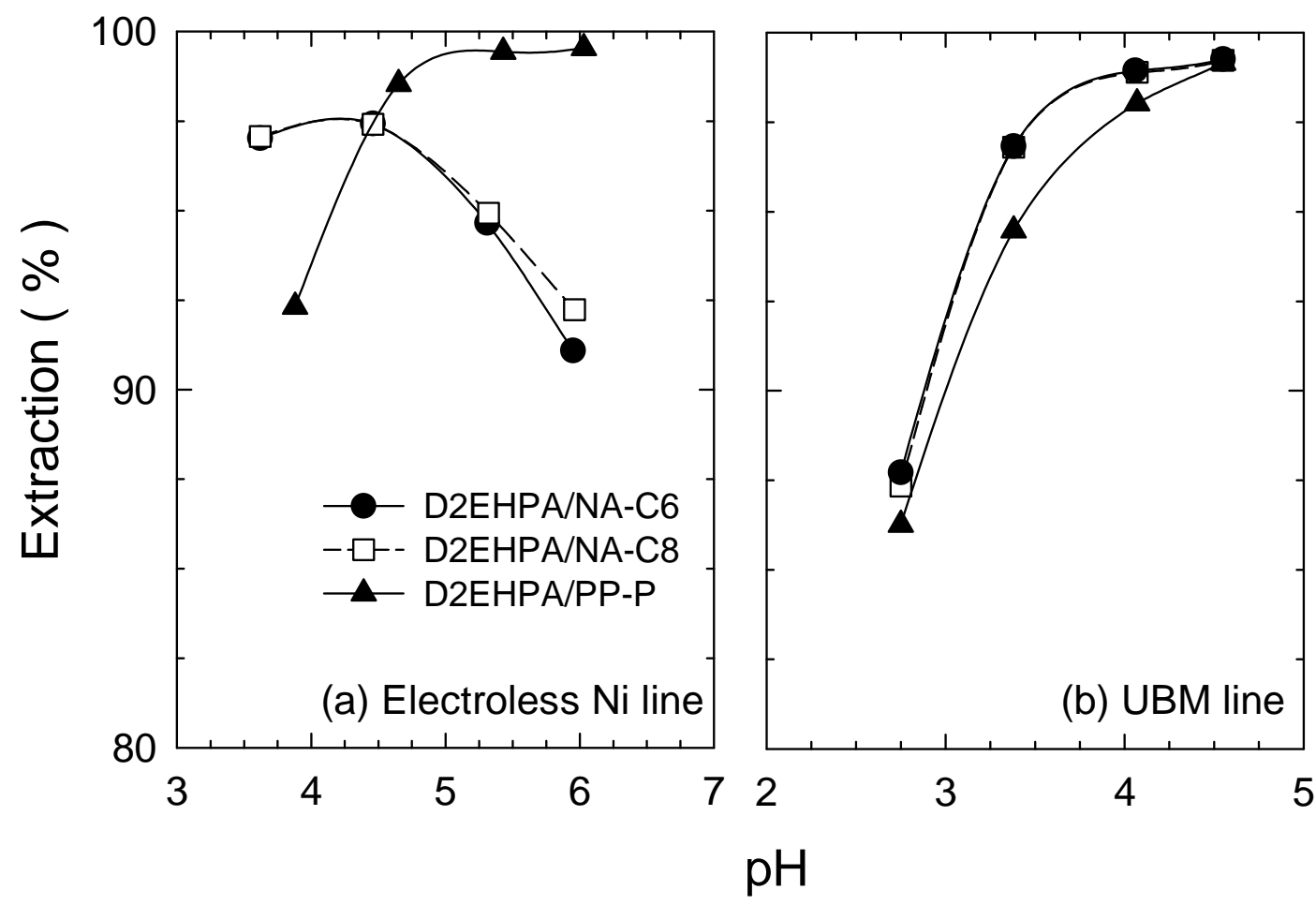

Figure 2. Effect of equilibrium $\mathrm{pH}$ on the extraction percentage of $\mathrm{Ni}$ from waste solutions of (a) the electroless $\mathrm{Ni}$ line and (b) the UBM line with D2EHPA/pyridine compounds; $[\overline{\mathrm{D} 2 \mathrm{EHPA}}]=$ $[\overline{\text { Pyridine Compounds }}]=0.5 \mathrm{~mol} / \mathrm{L}$.

The effect of the concentration of the mixed extractants was then studied using D2EHPA/NA-C6 and D2EHPA/NA-C8. In these cases, the concentrations of both extractants were varied from $0.3 \mathrm{~mol} / \mathrm{L}-0.6$ $\mathrm{mol} / \mathrm{L}$. The extraction percentages of $\mathrm{Ni}$ from the waste solutions of both lines increased with increasing concentration of the mixed extractant, and more than $96 \%$ of Ni is extracted with the mixed extractants of $0.6 \mathrm{~mol} / \mathrm{L}$ concentration from both waste solutions.

\subsection{Stripping of Ni from the loaded organic solution}

Stripping of $\mathrm{Ni}$ from the loaded organic solution was investigated. The waste solutions of both lines were first extracted with either D2EHPA/NA-C6 or D2EHPA/NA-C8 at an initial pH of 5.0. The resultant organic solutions were then scrubbed with $0.01 \mathrm{~mol} / \mathrm{L} \mathrm{H}_{2} \mathrm{SO}_{4}$ solution, and were stripped with $\mathrm{H}_{2} \mathrm{SO}_{4}$ solutions of $0.2-0.8 \mathrm{~mol} / \mathrm{L}$. Figure 3 shows the effect of $\mathrm{H}_{2} \mathrm{SO}_{4}$ concentration on the stripping percentage of $\mathrm{Ni}$. In both waste solutions and both mixed extractants, the maximum stripping percentage of $\mathrm{Ni}$ is obtained with $0.4 \mathrm{~mol} / \mathrm{L} \mathrm{H}_{2} \mathrm{SO}_{4}$ solution. In addition, the stripping percentage of $\mathrm{Ni}$ with $0.4 \mathrm{~mol} / \mathrm{L} \mathrm{H}_{2} \mathrm{SO}_{4}$ from the organic solution of Ni extracted with D2EHPA/NA-C8 is higher than that from D2EHPA/NA-C6. 
Okada et al. have investigated stripping of $\mathrm{Ni}$ from D2EHPA/nicotinic acid dodecyl ester and D2EHPA/isonicotinic acid dodecyl ester systems, and have reported the stripping efficiency of Ni was increased with $\mathrm{H}_{2} \mathrm{SO}_{4}$ concentration [2]. The difference in the stripping behavior with the present work may be due to the difference in the pyridine compounds used.

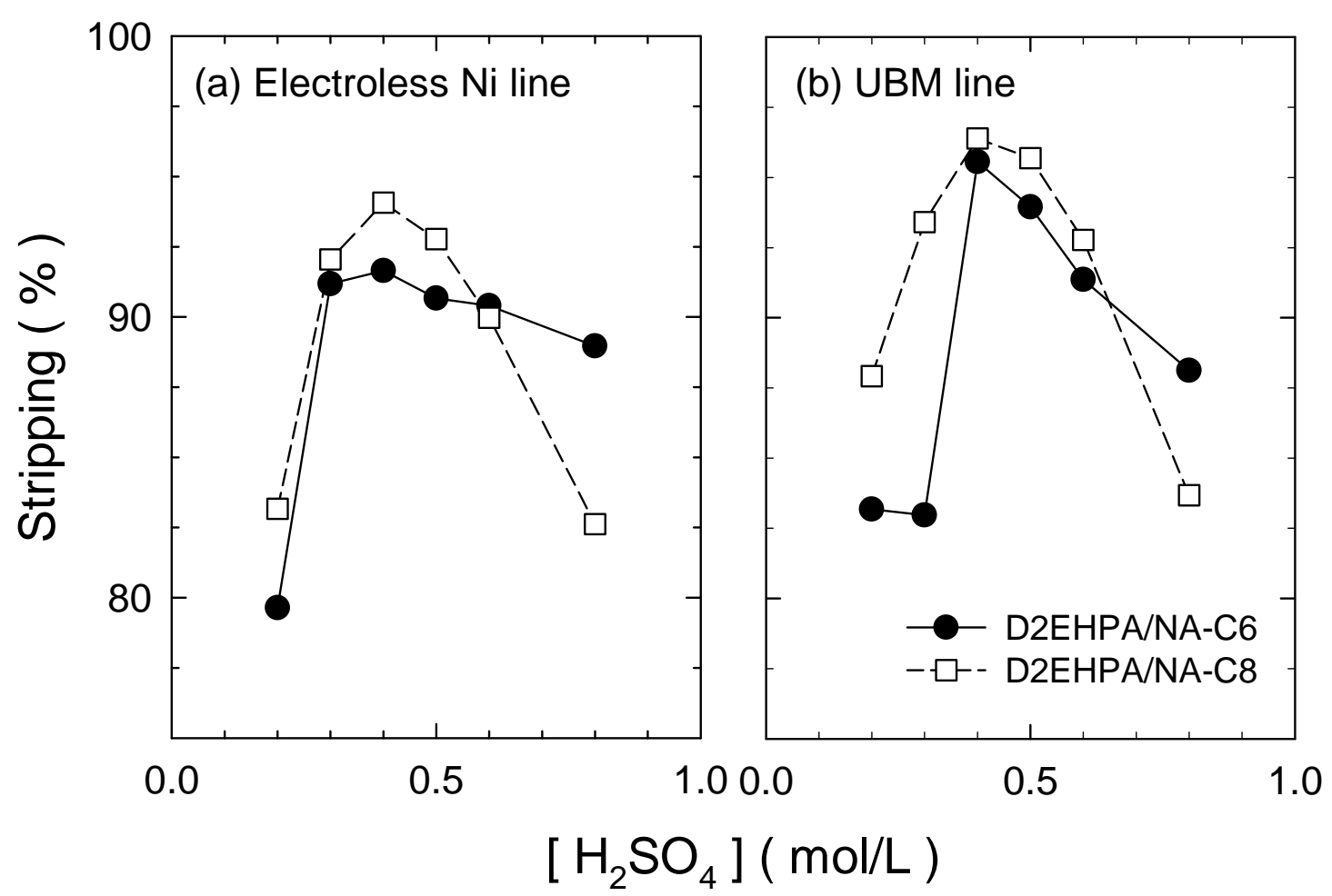

Figure 3. Effect of the concentration of $\mathrm{H}_{2} \mathrm{SO}_{4}$ solution on the stripping percentage of $\mathrm{Ni}$ from loaded organic solutions from (a) the electroless $\mathrm{Ni}$ line and (b) the UBM line; $[\overline{\mathrm{D} 2 \mathrm{EHPA}}]=$ $[\overline{\text { Pyridine Compounds }}]=0.6 \mathrm{~mol} / \mathrm{L}$.

\subsection{Reusability of the mixed extractants}

The reuse of the mixed extractants, during the iterative extraction-stripping processes, was also investigated. The aqueous feed solution $\mathrm{pH}$ was adjusted to $\mathrm{pH}$ 5.0, and the extraction was conducted with D2EHPA/NA-C6 (0.6 mol/L) or D2EHPA/NA-C8 $(0.6 \mathrm{~mol} / \mathrm{L})$. The organic solutions were then scrubbed with $0.01 \mathrm{~mol} / \mathrm{L} \mathrm{H}_{2} \mathrm{SO}_{4}$ solution, followed by stripping using $0.4 \mathrm{~mol} / \mathrm{L}$ of $\mathrm{H}_{2} \mathrm{SO}_{4}$. The stripped organic solution was then used for a second extraction - stripping process and so on. Figure 4 shows the extraction percentages of $\mathrm{Ni}$ with the mixed extractants, obtained by iterative processing. In the case of the D2EHPA/NA-C6 system, the extraction percentage of Ni decreased, significantly through the iterative processing, while that with D2EHPA/NA-C8 only slightly decreased. The difference in the reusability of the mixed extractant is caused by the loss of pyridine compounds which dissolved in the aqueous solution. D2EHPA/NA-C8 is therefore a suitable extraction system for the separation and recovery of $\mathrm{Ni}$ from the 
waste electroless Ni-P plating solution, although the NA-C8 content should be replenished to maintain extractability.

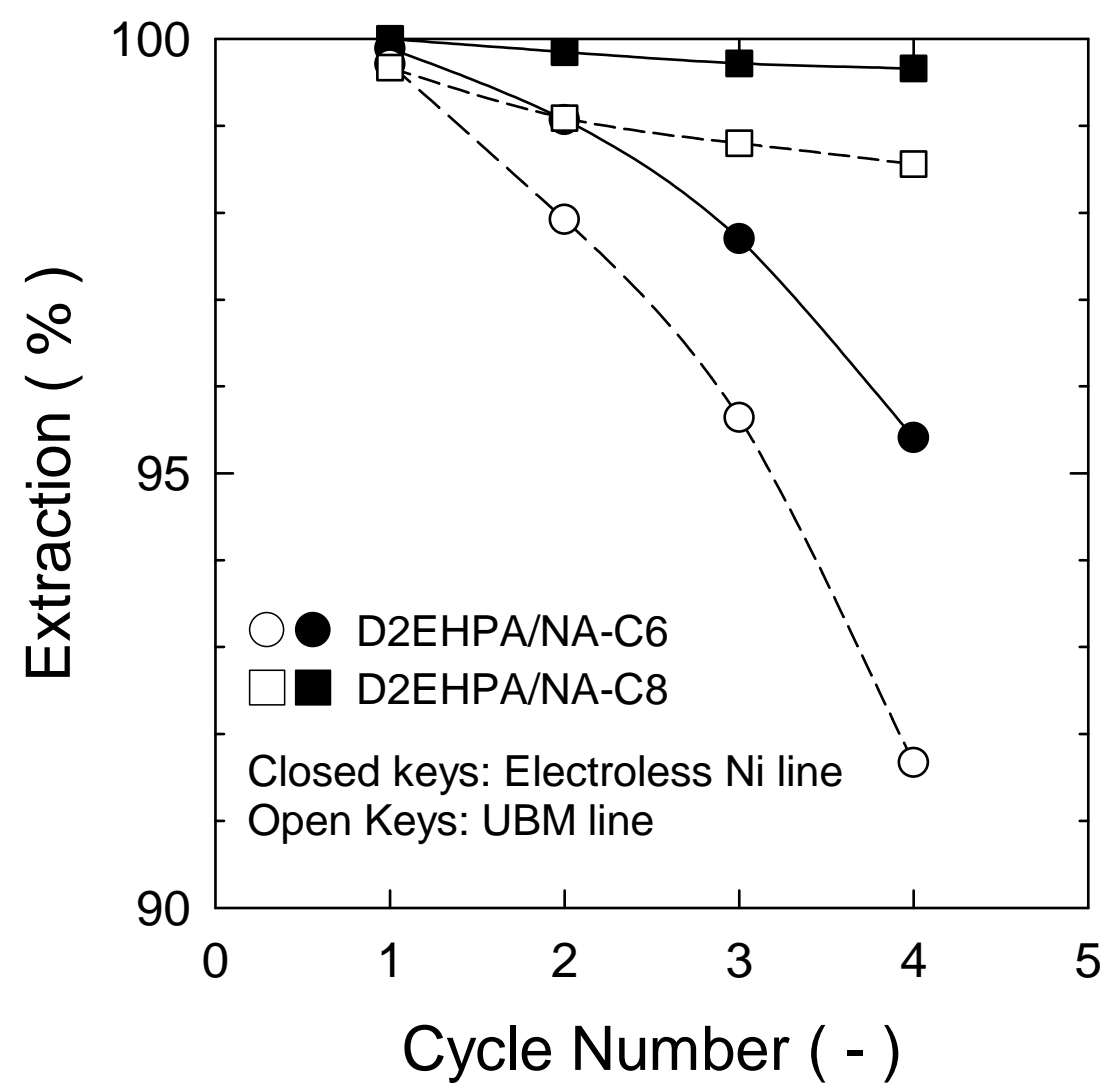

Figure 4. Effect of repeated processing on the extraction percentage of $\mathrm{Ni}$ with mixed extractants; $[\overline{\mathrm{D} 2 \mathrm{EHPA}}]=[\overline{\text { Pyridine Compounds }}]=0.6 \mathrm{~mol} / \mathrm{L}$.

The purity of the $\mathrm{Ni}$ in the strip liquor was finally investigated. Figure 5 shows the concentrations of $\mathrm{Ni}$ and $\mathrm{Na}$ in the stripping solution from D2EHPA/NA-C8 and the purity of Ni based on the contents of the metallic species. Other components present in the waste solution, apart from $\mathrm{Ni}$ and $\mathrm{Na}$, were not detected in the strip liquor. In both cases, $\mathrm{Na}$ is still contained in the strip liquor. In addition, the concentration of $\mathrm{Na}$ in the case of the UBM line is increased compared with the original waste solution due to the addition of $\mathrm{NaOH}$ solution to adjust the $\mathrm{pH}$ value. The purities of $\mathrm{Ni}$ are $81.2 \pm 1.9 \%$ for the electroless $\mathrm{Ni}$ line and $94.0 \pm 0.2 \%$ for the UBM line $(n=4$, error $=1 \sigma)$. The synergistic extraction system in the present work therefore provides a stable separation and recovery capability for Ni from the waste electroless Ni-P plating solution. Removal of Na from the stripping solution should be carefully conducted by optimizing the scrubbing conditions between extraction and stripping. 


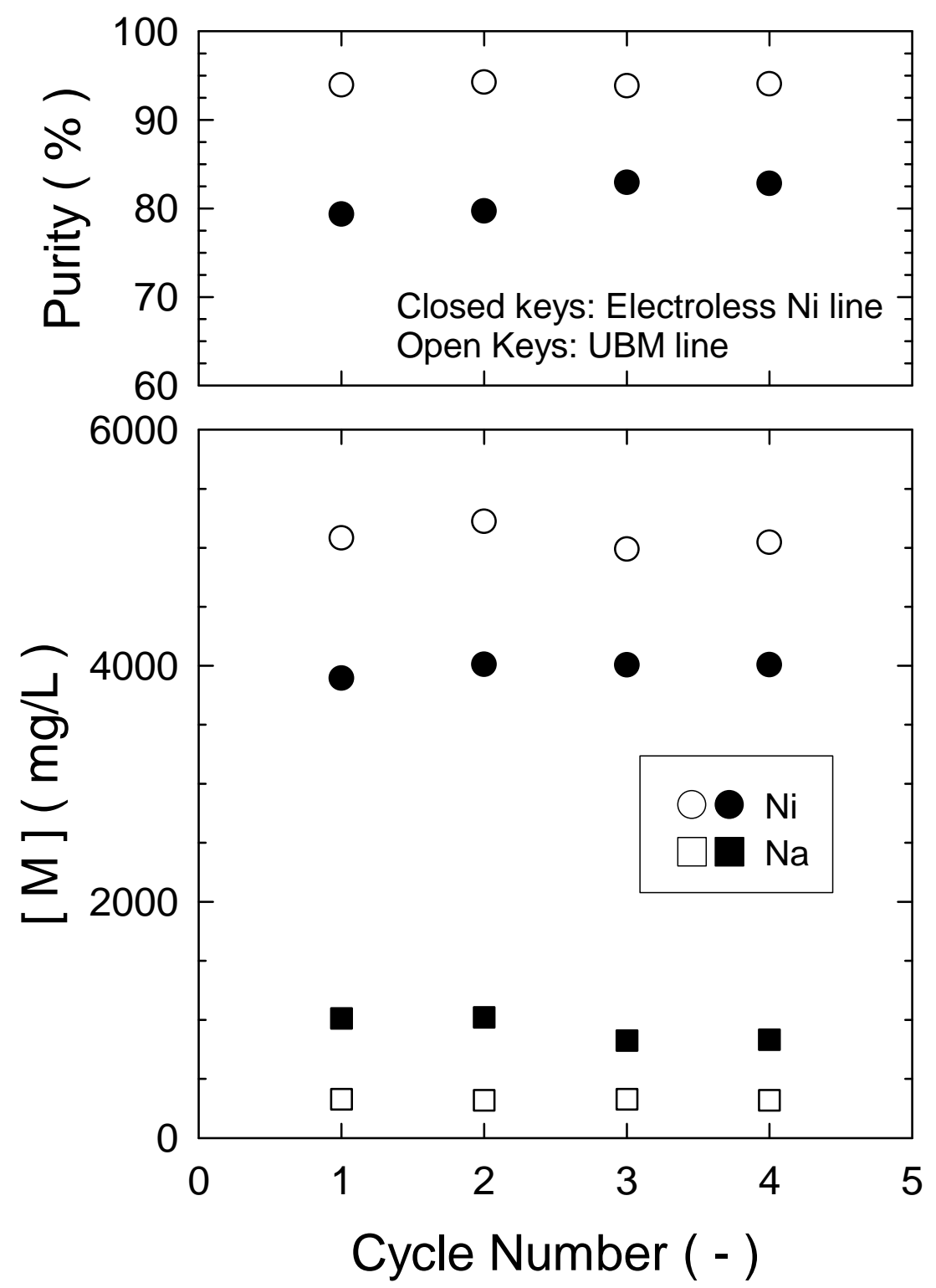

Figure 5. Effect of repeated processing on the concentration of $\mathrm{Ni}$ and $\mathrm{Na}$ and the purity of $\mathrm{Ni}$ with D2EHPA/NA-C8.

\section{Conclusion}

Separation and recovery of Ni from waste electroless Ni-P plating solutions has been investigated, with the following results.

(1) Extraction of $\mathrm{Ni}$ with D2EHPA alone is suppressed due to complexation reactions of $\mathrm{Ni}$ with phosphorous compounds coexisting in the waste solution. The extraction of $\mathrm{Ni}$ is dramatically improved when synergistic extraction is applied for all synergistic extraction systems.

(2) PP-P and NA-C6 are difficult to use in the process, because these are likely to dissolve in the 
aqueous phase.

(3) Almost quantitative stripping can be achieved with $0.4 \mathrm{~mol} / \mathrm{L} \mathrm{H}_{2} \mathrm{SO}_{4}$ solution.

(4) Stable extractability and stripping efficiency of Ni can be obtained with D2EHPA/NA-C8 during iterative processing. Removal of Na from the strip liquor should be carefully considered to improve the purity of the recovered $\mathrm{Ni}$.

\section{References}

1) M. Tanaka, H. Narita, Y. Saiki, Kagaku Kogaku Ronbunshu, 36, 201-206 (2010) (in Japanese).

2) M. Okada, S. Kimura, N. Murayama, J. Shibata, K. Otani, Resources Processing, 57, 109-113 (2010).

3) M. Tanaka, M. Maruyama, Y. Sato, Solvent Extr, Res. Dev., Jpn., 10, 51-63 (2003).

4) I. Komasawa, T. Otake, Y. Higaki, J. Inorg. Nucl. Chem., 43, 3351-3355 (1981).

5) T. Takahashi, T. Sato, Shigen-to-Sozai, 111, 787-792 (1995).

6) K. Ohto, T. Wada, M. Yano, H. Higashiyama, K. Inoue, Solvent Extr, Res. Dev., Jpn., 5, $43-50$ (1998). 